Agronomía Costarricense 37(2): 45-54. ISSN:0377-9424 / 2013

www.mag.go.cr/rev_agr/index.html www.cia.ucr.ac.cr

\title{
EVALUACIÓN DEL POTENCIAL FORRAJERO DE DIEZ CULTIVARES DE SOYA (Glycine max (L) Merr) EN VENEZUELA
}

\author{
Alexander Hernández ${ }^{1 / *}$, Robert Guerra*, Carlos Tobía**, Enrique Villalobos ${ }^{* * *}$ \\ Palabras clave: Forraje de soya, selección, biomasa, composición bromatológica. \\ Keywords: Soybeans forage, selection, biomass, chemical composition.
}

Recibido: 12/03/13

RESUMEN

Con el objetivo de evaluar y seleccionar cultivares de soya (Glycine max. (L) Merr) con fines de forraje, se llevó a cabo un experimento de un diseño en bloques completamente al azar con 6 repeticiones. Los materiales experimentales usados fueron 9 cultivares procedentes del banco de germoplasma de La Fundación DANAC y el cultivar CIGRAS-06 provenientes del Centro de Investigaciones de Granos y Semillas de la UCR. Las variables bajo estudio fueron las relacionadas con la arquitectura de la planta, producción de biomasa total en el estado reproductivo $R_{6}$, rendimiento de grano y algunas variables bromatológicas. Los análisis estadísticos mostraron diferencias significativas para las variables objeto de estudio. Los cultivares con mayores rendimientos de biomasa fueron CIGRAS-06, FP93-1935, FP93-1924 y FP906102 con $17245,13075,13066$ y 12587 kg.ha ${ }^{-1}$ de materia seca (MS). Los de mayores contenidos de proteína cruda (PC) en las vainas fueron FP9010205, FP93-1930 y FP92-1904, con 24,2, 23,7 y $21,0 \%$, respectivamente; y a nivel de las hojas los cultivares FP93-1935, FP92-4906 y FP90-7102 con $25,5,24,9$ y $23,0 \%$, respectivamente. Para la

\footnotetext{
1 Autor para correspondencia. Correo electrónico: ahernandez@ucla.edu.ve

* Decanato de Agronomía. Universidad Centroccidental "Lisandro Alvarado" (UCLA). Barquisimeto, Estado Lara, Venezuela.
}

Aceptado: 03/06/13

ABSTRACT
Evaluation of forage potential of ten
soybean cultivars (Glycine max (L) Merr)
in Venezuela. In order to evaluate and select
cultivars of soybean (Glycine max (L) Merr) for
forage purposes, an experiment was conducted
using a randomized complete block design
with 6 replications. The experimental materials
used were 9 cultivars from the germplasm bank
DANAC Foundation and cultivar CIGRAS-06
from the Research Center of Grain and Seeds
of the University of Costa Rica. The variables
studied were those related to plant architecture,
total biomass production in the reproductive
R6, grain yield and some bromatological
variables. Statistical analyzes showed significant
differences for the variables under study. The
cultivars with the highest biomass yields were
CIGRAS-06, FP93-1935, FP93-1924 and FP90-
6102, with 17245, 13075, 13066 and 12587 kg.ha
1 of dry matter (DM). The higher content of
crude protein (CP) in the pods were FP90-
10205 , FP93-1930 and FP92-1904, with 24.2,
23.7 and 21.0\%, respectively. And at leaf-level,
cultivars FP93-1935, FP92-4906 and FP90-7102
with 25.5, 24.9 and 23.0\%, respectively. For grain
** Decanato de Ciencias Veterinarias. UCLA,
*** Centro de Investigaciones en Granos y Semillas
Costa Rica.

\section{ABSTRACT} soybean cultivars (Glycine max (L) Merr) in Venezuela. In order to evaluate and select cultivars of soybean (Glycine $\max (\mathrm{L})$ Merr) for forage purposes, an experiment was conducted using a randomized complete block design with 6 replications. The experimental materials used were 9 cultivars from the germplasm bank DANAC Foundation and cultivar CIGRAS-06 of the University of Costa Rica. The variables studied were those related to plant architecture, total biomass production in the reproductive R6, grain yield and some bromatological variables. Statistical analyzes showed significant differences for the variables under study. The cultivars with the highest biomass yields were CIGRAS-06, FP93-1935, FP93-1924 and FP906102, with 17245, 13075, 13066 and 12587 kg.ha ${ }^{-}$ 1 of dry matter (DM). The higher content of crude protein (CP) in the pods were FP9010205, FP93-1930 and FP92-1904, with 24.2, cultivars FP93-1935, FP92-4906 and FP90-7102 with $25.5,24.9$ and $23.0 \%$, respectively. For grain 
producción de grano mostraron alto potencial los cultivares CIGRAS-06, FP93-1924, FP90-6102 y FP90-6103, al superar los 3500 kg.ha-1.

\section{INTRODUCCIÓN}

En Venezuela, la necesidad de producir soya está dada por su gran demanda. Su producción al principio estuvo limitada por la falta de variedades adaptadas al trópico, problema que fue solventado por las investigaciones de diversas instituciones llevadas a cabo mediante programas de mejoramiento genético y selección de cultivares con los cuales se logró producir soya en algunas regiones del país de manera satisfactoria obteniéndose rendimientos de grano entre los 1600 y $2500 \mathrm{~kg} \cdot \mathrm{ha}^{-1}$. Anualmente en el país se consume más de $1 \times 10^{6}$ ton de grano de soya, casi en su totalidad importada. Para el año 2011 apenas se produjeron $0,05 \times 10^{6}$ ton con un rendimiento de $1360 \mathrm{~kg} \cdot \mathrm{ha}^{-1}$. Esa marcada dependencia de las importaciones vulnera la seguridad alimentaria y evidencia la necesidad de impulsar el cultivo de la soya en el país (Fedeagro 2011).

Las necesidades de consumo de soya, para la producción de alimentos concentrados para animales, alcanza alrededor de 200000 ton.año $^{-1}$, las cuales son importadas en su totalidad. De intentarse producir en el país toda la soya que se importa, se estima que sería necesario cultivar alrededor de 600000 ha con un rendimiento promedio nacional de $1500 \mathrm{~kg} \cdot \mathrm{ha}^{-1}$ aproximadamente (Solórzano et ál. 2005).

Los altos costos de la producción de leche y carne en el trópico, debido principalmente a los altos precios de las materias primas importadas utilizadas en la elaboración de los alimentos balanceados, es la causa principal de la baja rentabilidad que actualmente afecta a esta actividad. Una alternativa viable para reducir los costos de alimentación en los sistemas tropicales de producción de leche y carne, los cuales requieren production, cultivars showing high potential were CIGRAS-06, FP93-1924, FP90-6102 and FP906103 , exceeding $3500 \mathrm{~kg} \cdot \mathrm{ha}^{-1}$.

de elevadas cantidades de energía y proteínas, es producir forrajes de alta calidad que permitan al productor reducir la cantidad de alimentos balanceados (concentrados) en las raciones de los animales (Tobía et ál. 2006).

El forraje de soya cosechado en el estado $R_{6}$, en el cual una vaina en cualquiera de los 4 nudos superiores del tallo principal contiene una semilla verde que llena la cavidad de dicha vaina, es comparable con la alfalfa (Hintz y Albretcht 1994), la cual es considerada un forraje de excelente calidad nutricional por contener altos contenidos de proteína y bajos contenidos de fibra (Weiss y Shockey 1991). Adicionalmente, el forraje de soya presenta un mayor contenido de energía que la alfalfa, debido a la mayor cantidad de grasas (extracto etéreo) que contiene la semilla inmadura de la soya en este estado de desarrollo (Tobía 2004).

Después del estado $\mathrm{R}_{5}$ la acumulación de materia seca y nutrientes de las hojas, pecíolos y tallos, se hace máxima y comienza a redistribuirse de estos órganos a la semillas. Poco después de $\mathrm{R}_{6}$ ocurre un periodo de rápida acumulación de materia seca en la semilla durante el cual alcanza el $80 \%$ de su masa seca (Harper 1974).

El cultivo de la soya se destaca principalmente por la producción de grano; sin embargo, se conoce del buen comportamiento de algunos materiales con respecto a la acumulación de biomasa. Se señalan producciones de 19 toneladas de forraje verde por hectárea en condiciones adversas en Costa Rica (Tobía y Villalobos 2004) y de 30 ton $^{-1}$ promedio en condiciones favorables en Venezuela (Tobía et ál. 2007) cuando se cosecha el forraje de soya en estado $\mathrm{R}_{6}$.

El forraje de soya posee una excelente composición nutricional: a) el contenido de 
proteína cruda (PC) varía entre 12,0 y $20,2 \%$ que depende de los niveles de suplencia hídrica (Tobía 2011); b) las concentraciones de fibra neutra detergente (FND) se aproximan a 48,0\% del total de la MS y los valores de degradabilidad ruminal de la MS se encuentran cercanos al 75\% (Sequera 2009). Estos parámetros son considerados satisfactorios para la nutrición animal.

El valor nutricional del forraje está limitado por su composición bromatológica y su digestibilidad. El uso de esta composición bromatológica como indicador de la calidad de un forraje es muy común y es importante que tales patrones analíticos de calidad, reflejen los factores reales que determinen la composición y calidad del forraje (Van Soest 1994).

Según lo anterior este estudio tuvo como objetivo evaluar y seleccionar cultivares de soyas basado en características de la arquitectura de la planta, biomasa, composición bromatológica y producción de grano de 10 cultivares adaptados a condiciones tropicales con potencial para la producción de forraje.

\section{MATERIALES Y MÉTODOS}

El experimento fue desarrollado en los terrenos del núcleo Tarabana perteneciente al Decanato de Agronomía de la Universidad Centroccidental "Lisandro Alvarado" UCLA, Cabudare, estado Lara, Venezuela $\left(10^{\circ} 01^{\prime} 25\right.$ " $\mathrm{N}$ y $69^{\circ} 16^{\prime} 44^{\prime \prime}$ O). Esta zona se caracteriza por presentar una temperatura media anual de $25,3^{\circ} \mathrm{C}$, precipitación de $812,6 \mathrm{~mm}$ y altura de $510 \mathrm{msnm}$. Esto corresponde con una zona de vida en transición entre un Bosque seco tropical (Bst) y Bosque seco (BS) (Rodríguez 2003).

Se emplearon 9 líneas provenientes del Banco de Germoplasma de la Fundación para la Investigación Agrícola DANAC y un cultivar proveniente de la Universidad de Costa Rica CIGRAS-06, el cual fue utilizado como testigo. El cultivar CIGRAS-06 es el producto del cruzamiento entre las variedades Padre y Duocrop adaptada al trópico (Villalobos y Camacho 2003). Estos mismos autores indican que es un cultivar de crecimiento semi-determinado que reduce el crecimiento y el volcamiento en condiciones de excesiva precipitación. Este material fue seleccionado por su alta productividad de grano y forraje en las diferentes localidades de Costa Rica y Venezuela (Villalobos y Camacho 1999, Tobía et ál. 2007).

El diseño utilizado fue de bloques completamente aleatorizados con 6 repeticiones. Cada unidad experimental formada por 3 hileras de 5 $\mathrm{m}$ de largo separada a 0,5 m entre ellas. Para la siembra se distribuyó en cada hilera la cantidad de semillas correspondientes por cada tratamiento a un promedio de 15 plantas por metro lineal. Las semillas se inocularon antes de la siembra con cepas de Bradyrizobium japonicum. Para el control de malezas se aplicó el herbicida preemergente Prowl 400 Ec (Pendimetalín) en dosis de 3 1.ha $^{-1}$. Los posteriores controles se hicieron de forma manual. La fertilización se realizó aplicada en bandas después de la siembra con $400 \mathrm{~kg} \cdot \mathrm{ha}^{-1}$ de la fórmula 10-20-20 cp.

Las muestras para analizar la masa fresca y seca de la planta entera de soya y sus diferentes partes, fueron tomadas cuando las plantas alcanzaran el estado de desarrollo $\mathrm{R}_{6}$ (Fehr y Caviness 1980). Se seleccionaron de cada parcela puntos de recolección en donde se cosecharon las plantas distribuidas en un metro. Con estas muestras se determinaron las variables de campo y de laboratorio.

En campo se midieron: altura de la primera vaina (APV), número de ramas (NR), número de semillas por vainas (NSV), número de vainas por planta (NVP), altura de planta (AP), masa de 100 semillas (M100S), rendimiento de grano por planta (RGp), rendimiento por hectárea $\left(\right.$ Rend.ha ${ }^{-1}$ y masa fresca y seca de las hojas, tallos y vainas.

En el Laboratorio de Nutrición Animal del Decanato de Ciencias Veterinarias se midieron las variables bromatológicas. Luego de haber separado de las plantas las hojas, vainas y tallos. Cada muestra fue secada en un horno con flujo de aire a $60^{\circ} \mathrm{C}$ por $48 \mathrm{~h}$ y luego se molieron en un molino Willey $\mathrm{N}^{\circ} 3$ tipo estándar en una malla de 
$1 \mathrm{~mm}$ de diámetro. A continuación se procedió a guardar las muestras en bolsas herméticas previamente identificadas y proceder a determinar un análisis proximal por duplicado de los contenidos de materia seca (MS) y proteína cruda (PC) mediante las técnicas descritas por la AOAC (1990) y la concentración de la fibra neutro detergente (FND) por el procedimiento descrito por Van Soest (1991).

Todas las variables fueron objeto de un análisis de varianza, en un modelo lineal, para lo cual el efecto de los bloques se consideró aleatorio. Los cálculos se realizaron con el paquete estadístico SAS-JMP-7 y para la comparación de medias se usó la prueba de mínima diferencia significativa (MDS) al 5\% de probabilidad. Se construyó una matriz de correlación entre los datos estandarizados que permitió el análisis de componentes principales (ACP) por medio del programa InfoGen (Balzarini y Di Rienzo 2012).

\section{RESULTADOS Y DISCUSIÓN}

\section{Características agronómicas}

Las variables altura de planta, altura de la primera vaina y número de ramas, relacionadas con la arquitectura de la planta, mostraron diferencias significativas $(p<0,05)$ entre los cultivares (Cuadro 1). Se buscan cultivares con mayor altura de planta, que comience a producir vainas lo más bajo posible a partir de los $12 \mathrm{~cm}$ y que tengan en promedio más de 2,5 ramas. Para la altura de planta los cultivares FP90-6103, CIGRAS-06 y FP93-1935 se comportan de manera similar con valores por encima de $71,74 \mathrm{~cm}$. Los

Cuadro 1. Medias de los cultivares para las variables relacionadas con la arquitectura de la planta y los componentes del rendimiento.

\begin{tabular}{lllllllc}
\hline Cultivares & $\begin{array}{c}\text { AP } \\
(\mathrm{cm})\end{array}$ & $\begin{array}{c}\text { ApV } \\
(\mathrm{cm})\end{array}$ & NR & NVP & NSV & $\begin{array}{c}\text { M100S } \\
(\mathrm{g})\end{array}$ & $\begin{array}{c}\text { RGp } \\
(\mathrm{g})\end{array}$ \\
\hline CIGRAS-06 & $76,06 \mathrm{ab}$ & $16,56 \mathrm{c}$ & $2,64 \mathrm{~b}$ & $48,17 \mathrm{ab}$ & $2,44 \mathrm{a}$ & $20,03 \mathrm{a}$ & $20,05 \mathrm{a}$ \\
FP92-1904 & $67,36 \mathrm{bcd}$ & $20,37 \mathrm{~b}$ & $1,92 \mathrm{~b}$ & $51,27 \mathrm{ab}$ & $1,55 \mathrm{f}$ & $18,05 \mathrm{ab}$ & $11,48 \mathrm{bc}$ \\
FP93-1935 & $71,74 \mathrm{abc}$ & $20,13 \mathrm{~b}$ & $2,43 \mathrm{~b}$ & $48,75 \mathrm{ab}$ & $1,77 \mathrm{e}$ & $14,96 \mathrm{~cd}$ & $12,49 \mathrm{bc}$ \\
FP90-7102 & $67,17 \mathrm{bcd}$ & $18,91 \mathrm{bc}$ & $2,37 \mathrm{~b}$ & $51,31 \mathrm{ab}$ & $2,12 \mathrm{bc}$ & $15,31 \mathrm{bcd}$ & $9,99 \mathrm{bc}$ \\
FP93-1924 & $68,01 \mathrm{bcd}$ & $20,53 \mathrm{~b}$ & $2,74 \mathrm{~b}$ & $69,60 \mathrm{a}$ & $1,89 \mathrm{de}$ & $14,67 \mathrm{~cd}$ & $15,17 \mathrm{ab}$ \\
FP90-10205 & $60,02 \mathrm{~d}$ & $16,14 \mathrm{~cd}$ & $2,75 \mathrm{~b}$ & $40,22 \mathrm{~b}$ & $2,08 \mathrm{bcd}$ & $16,16 \mathrm{bc}$ & $11,74 \mathrm{bc}$ \\
FP92-4906 & $62,13 \mathrm{~cd}$ & $19,62 \mathrm{bc}$ & $2,38 \mathrm{~b}$ & $46,98 \mathrm{ab}$ & $1,81 \mathrm{e}$ & $13,18 \mathrm{~d}$ & $6,15 \mathrm{c}$ \\
FP90-6103 & $80,90 \mathrm{a}$ & $24,94 \mathrm{a}$ & $2,03 \mathrm{~b}$ & $57,33 \mathrm{ab}$ & $1,93 \mathrm{cde}$ & $15,10 \mathrm{~cd}$ & $14,37 \mathrm{ab}$ \\
FP93-1930 & $62,22 \mathrm{~cd}$ & $17,03 \mathrm{bc}$ & $2,27 \mathrm{~b}$ & $59,85 \mathrm{ab}$ & $1,79 \mathrm{e}$ & $15,53 \mathrm{bcd}$ & $11,38 \mathrm{bc}$ \\
FP90-6102 & $58,55 \mathrm{~d}$ & $12,51 \mathrm{~d}$ & $3,77 \mathrm{a}$ & $64,42 \mathrm{ab}$ & $2,23 \mathrm{ab}$ & $14,13 \mathrm{~cd}$ & $15,14 \mathrm{ab}$ \\
\hline Promedio & 67,42 & 18,67 & 2,53 & 53,79 & 1,96 & 15,71 & 12,79 \\
\hline C.V.(\%) & 12,28 & 14,68 & 25,73 & 36,28 & 7,90 & 13,24 & 15,35 \\
\hline
\end{tabular}

$\mathrm{AP}=$ Altura de planta, ApV=Altura de la primera vaina, NR=Número de ramas, NVP=número de vainas por planta; NSV=Número de semillas por vainas; M100S=Masa de 100 semillas; RGp=Rendimiento del grano por planta. Medias con la misma letra en cada columna no difieren de acuerdo con la prueba de MDS al $5 \%$. 
cultivares con las primeras vainas más bajas fueron: FP90-6102, FP90-10205, CIGRAS-06, FP93-1930, FP90-7102 y FP92-4906 con valores menores a 19,62 cm. El número de ramas por planta varió entre 3,8 y 1,9. Los cultivares que mostraron más de 2,5 ramas por planta en promedio fueron: FP90-6102, FP90-10205, FP93-1924 y CIGRAS-06. Plantas con altura de la primera vaina por encima de $12 \mathrm{~cm}$ facilitan la cosecha mecánica y disminuyen la perdida de grano durante la recolección. No es deseable una mayor producción de ramas en plantas para ser cosechadas mecánicamente, porque muchas pueden presentar en los nudos inferiores, vainas más cercanas al suelo que durante la cosecha incrementan las pérdidas de material forrajero y granos (Norman 1983).

En cuanto a las variables del rendimiento y sus componentes (Cuadro 1) se encontraron diferencias $(\mathrm{p}<0,05)$ entre los cultivares. El número vainas por planta varió entre 40,22 y 69,60 y la mayoría de los cultivares mostraron valores intermedios. Los cultivares con mayor número de semillas por vainas fueron CIGRAS $06 \mathrm{y}$ FP90-6102 con 2,44 y 2,23 semillas/vaina, respectivamente. Estos resultados inducen a pensar que en los tratamientos donde se obtuvieron los mayores valores se presentaron mayor proporción de vainas con 3 semillas. Gazzoni (1995) indica que existe predominancia en vainas con 2 semillas en la mayoría de los cultivares de soya. En relación con la masa de 100 semillas, el cultivar CIGRAS 06 y FP92-1904 muestran las semillas más grandes con masa de 20,03 y 18,05 g., respectivamente.

Evaluaciones realizadas por Maduro et ál. (2012) con el cultivar CIGRAS-06 indican masa de 100 semillas entre 12,6 y 21,4 g., cuando se le suministraron 17 y $107 \%$ de los requerimientos hídricos al cultivo, respectivamente. Por su parte, Norman 1983 y Acevedo 1998, señalan valores entre 12,0 y 19,0 g., donde las diferencias en la masa y tamaño de los granos es multifactorial (germoplasma, condiciones agroclimáticas entre otros).
En relación con el rendimiento del grano por planta se obtuvieron valores extremos entre 20,05 y 6,15 g. Los más altos valores se obtuvieron con los cultivares CIGRA-06, FP93-1924, FP90-6102 y FP90-6103 que superaron al resto de los cultivares.

En general, los cultivares evaluados difieren en su comportamiento agronómico y ninguno supera al CIGRAS-06 el cual cumple con el porte de planta requerido para ser considerado un material doble propósito (productor de forraje y grano).

Los cultivares mostraron diferencias $(p<0,05)$ para las variables masa fresca y seca en todas las fracciones de la parte aérea de la planta. Se prefieren cultivares con mayores masas en vainas y hojas ya que almacenan mayor contenido de proteína cruda, además de contener menos fibra (Tobía y Villalobos 2004). Por el contrario se buscan cultivares con menos masa de tallo por estar relacionado con un alto contenido de FND y bajas concentraciones proteína.

En el Cuadro 2 se puede observar que el cultivar CIGRAS-06 se destaca por su mayor producción de biomasa, tanto de vainas, hojas y tallo, como era de esperar dado el mayor porte de este cultivar. Para la variable masa fresca de vainas (MFv), además del CIGRAS-06 el cual duplica el valor promedio, se destacan los cultivar FP90-6102, FP93-1924 y FP93-1935 con valores $241,0,226,7$ y 221,2 g, respectivamente. En relación con la masa fresca de las hojas (MFh) resaltan los cultivares FP93-1935, FP93-1924 y FP90-6102 con valores de 516,4, 407,6 y 404,9 g, respectivamente. De igual manera, la masa fresco del tallo (MFt) el cultivar CIGRAS-06 supera al resto de los cultivares con un valor de 442,0 g. Los cultivares FP93-1935, FP93-1924, FP906103 y FP90-6102 muestras valores por encima del promedio (Cuadro 2).

De igual manera para masa seca los cultivares siguen el mismo comportamiento mostrado en masa fresca, en donde el CIGRAS-06 supera ampliamente en MSv y MSt al resto de los materiales y es similar para MSh al FP93-1935, FP931924 y FP90-6102. 
Cuadro 2. Media de los cultivares para las variables relacionadas con la masa fresca y seca de las fracciones de la parte aérea de la planta.

\begin{tabular}{lllllll}
\hline Cultivares & MFv $(\mathrm{g})$ & MFh $(\mathrm{g})$ & MFt $(\mathrm{g})$ & MSv $(\mathrm{g})$ & MSh $(\mathrm{g})$ & MSt $(\mathrm{g})$ \\
\hline CIGRAS-06 & $465,5 \mathrm{a}$ & $348,4 \mathrm{bcd}$ & $442,0 \mathrm{a}$ & $135,2 \mathrm{a}$ & $141,0 \mathrm{ab}$ & $137,7 \mathrm{a}$ \\
FP92-1904 & $159,5 \mathrm{bcd}$ & $293,7 \mathrm{cde}$ & $188,6 \mathrm{~cd}$ & $47,1 \mathrm{bc}$ & $108,1 \mathrm{~cd}$ & $68,4 \mathrm{bcd}$ \\
FP93-1935 & $221,2 \mathrm{~b}$ & $516,4 \mathrm{a}$ & $315,8 \mathrm{~b}$ & $56,8 \mathrm{bc}$ & $155,9 \mathrm{a}$ & $101,2 \mathrm{~b}$ \\
FP90-7102 & $145,3 \mathrm{bcd}$ & $237,6 \mathrm{de}$ & $171,2 \mathrm{~cd}$ & $47,8 \mathrm{bc}$ & $95,6 \mathrm{~d}$ & $66,0 \mathrm{bcd}$ \\
FP93-1924 & $226,7 \mathrm{~b}$ & $407,6 \mathrm{ab}$ & $285,8 \mathrm{bc}$ & $72,1 \mathrm{~b}$ & $146,1 \mathrm{a}$ & $95,4 \mathrm{~b}$ \\
FP90-10205 & $109,1 \mathrm{~cd}$ & $246,5 \mathrm{de}$ & $175,1 \mathrm{~cd}$ & $35,1 \mathrm{bc}$ & $85,7 \mathrm{~d}$ & $60,5 \mathrm{~cd}$ \\
FP92-4906 & $79,1 \mathrm{~d}$ & $201,6 \mathrm{e}$ & $110,0 \mathrm{~d}$ & $31,7 \mathrm{c}$ & $86,5 \mathrm{~d}$ & $45,4 \mathrm{~d}$ \\
FP90-6103 & $215,1 \mathrm{bc}$ & $268,2 \mathrm{de}$ & $244,2 \mathrm{bc}$ & $69,6 \mathrm{~b}$ & $101,4 \mathrm{~d}$ & $84,0 \mathrm{bc}$ \\
FP93-1930 & $171,9 \mathrm{bcd}$ & $315,3 \mathrm{bcde}$ & $217,3 \mathrm{bcd}$ & $51,9 \mathrm{bc}$ & $111,8 \mathrm{bcd}$ & $73,2 \mathrm{bcd}$ \\
FP90-6102 & $241,0 \mathrm{~b}$ & $404,9 \mathrm{abc}$ & $259,8 \mathrm{bc}$ & $73,7 \mathrm{~b}$ & $140,7 \mathrm{abc}$ & $87,7 \mathrm{bc}$ \\
Promedio & 203,4 & 324,0 & 241,0 & 62,1 & 117,3 & 82,0 \\
\hline C.V.(\%) & 20,4 & 13,1 & 17,7 & 19,5 & 10,2 & 14,1 \\
\hline
\end{tabular}

$\mathrm{MFv}=$ Masa fresca vainas, $\mathrm{MFh}=$ Masa fresca hojas, $\mathrm{MFt}=$ Masa fresca tallos, $\mathrm{MSv}=$ Masa seca vainas, MSh=Masa seca hojas y MSt=Masa seca tallos. Medias con la misma letra en cada columna no difieren de acuerdo con la prueba de MDS al 5\%.

\section{Características bromatológicas}

El análisis bromatológico es un factor esencial para valorar el potencial nutricional de un alimento, así como su poder productivo, pues este se determina a través de análisis químicos cuantitativos en el laboratorio. En el Cuadro 3 se muestran las medias para proteína cruda (PC). En las vainas se aprecia un promedio de $20,2 \%$ entre los cultivares. El grupo de mayor contenido estuvo conformado por los cultivares FP90-10205, FP93-1930 y FP92-1904 con valores que superan al 21\%. En la fracción de las hojas se obtuvo un promedio de 20,6\% de proteína cruda y resaltan por su mayor porcentaje los cultivares FP93-1935, FP92-4906 y FP90-7102. A nivel de los tallos los cultivares mostraron valores entre 5,4 y $7,6 \%$, con una media de $6,5 \%$.

Tobía (2011) señala 23,5; 19,7 y 6,6\% de PC en vainas, hojas y tallos, respectivamente para el cultivar CIGRAS-06, los cuales fueron superiores en relación con las vainas y similares en hojas y tallos. Estas concentraciones de PC se obtuvieron cuando se le suministraron el $100 \%$ de los requerimientos hídricos al forraje de soya en estado $R_{6}$. Los porcentajes de fibra neutro detergente (FND) de las fracciones en los cultivares mostraron diferencias $(p<0,05)$. En las hojas fueron superiores y similares en sí, los cultivares CIGRAS-06, FP903-1924, FP93-1935 y FP924906, con valores por encima del 41,8\%. En la fracción de las vainas los cultivares FP92-4906, FP90-7102, FP90-6103, FP93-1935 y FP90-6102 superan al resto de los cultivares, con valores por encima de 47,3\%. En los tallos tienen mayor contenido los cultivares FP93-1935, FP90-10205 y FP90-6103, con valores que superan el 67,5\%.

En el Cuadro 4 se aprecian los valores de los rendimientos por hectárea de biomasa fresca y seca y del grano donde los mayores valores lo obtuvo el cultivar CIGRAS-06 con 52 332, 17245 
Cuadro 3. Media de los cultivares para las variables de proteína cruda (PC) y de fibra neutra detergente (FND) de las fracciones de la parte aérea de la planta.

\begin{tabular}{lllllll}
\hline \multicolumn{1}{c}{ Cultivares } & $\begin{array}{c}\text { PCv } \\
(\%)\end{array}$ & $\begin{array}{c}\text { PCh } \\
(\%)\end{array}$ & $\begin{array}{c}\text { PCt } \\
(\%)\end{array}$ & $\begin{array}{c}\text { FNDv } \\
(\%)\end{array}$ & $\begin{array}{c}\text { FNDh } \\
(\%)\end{array}$ & $\begin{array}{c}\text { FNDt } \\
(\%)\end{array}$ \\
\hline CIGRAS-06 & $19,7 \mathrm{~cd}$ & $18,2 \mathrm{def}$ & $6,5 \mathrm{ab}$ & $45,3 \mathrm{bcd}$ & $46,5 \mathrm{a}$ & $65,8 \mathrm{bc}$ \\
FP92-1904 & $21,0 \mathrm{bc}$ & $21,3 \mathrm{~cd}$ & $6,9 \mathrm{ab}$ & $43,6 \mathrm{~cd}$ & $31,5 \mathrm{~cd}$ & $63,4 \mathrm{c}$ \\
FP93-1935 & $19,2 \mathrm{~cd}$ & $25,5 \mathrm{a}$ & $5,9 \mathrm{ab}$ & $48,5 \mathrm{ab}$ & $41,8 \mathrm{ab}$ & $71,4 \mathrm{a}$ \\
FP90-7102 & $18,6 \mathrm{~cd}$ & $23,0 \mathrm{abc}$ & $6,3 \mathrm{ab}$ & $50,2 \mathrm{a}$ & $41,9 \mathrm{ab}$ & $64,7 \mathrm{bc}$ \\
FP93-1924 & $19,0 \mathrm{~cd}$ & $17,2 \mathrm{f}$ & $5,4 \mathrm{~b}$ & $47,7 \mathrm{abc}$ & $44,2 \mathrm{a}$ & $67,6 \mathrm{abc}$ \\
FP90-10205 & $24,2 \mathrm{a}$ & $22,0 \mathrm{bc}$ & $7,1 \mathrm{ab}$ & $42,7 \mathrm{~d}$ & $33,5 \mathrm{~cd}$ & $69,2 \mathrm{ab}$ \\
FP92-4906 & $18,5 \mathrm{~cd}$ & $24,9 \mathrm{ab}$ & $7,6 \mathrm{a}$ & $50,4 \mathrm{a}$ & $41,8 \mathrm{ab}$ & $64,1 \mathrm{c}$ \\
FP90-6103 & $20,6 \mathrm{c}$ & $17,4 \mathrm{ef}$ & $6,3 \mathrm{ab}$ & $48,7 \mathrm{ab}$ & $37,2 \mathrm{bc}$ & $67,5 \mathrm{abc}$ \\
FP93-1930 & $23,7 \mathrm{ab}$ & $15,7 \mathrm{f}$ & $5,9 \mathrm{ab}$ & $45,5 \mathrm{bcd}$ & $30,4 \mathrm{~d}$ & $65,7 \mathrm{bc}$ \\
FP90-6102 & $17,1 \mathrm{~d}$ & $20,6 \mathrm{cde}$ & $6,8 \mathrm{ab}$ & $47,3 \mathrm{abc}$ & $36,7 \mathrm{bc}$ & $64,3 \mathrm{bc}$ \\
\hline Promedio & 20,2 & 20,6 & 6,5 & 47,0 & 38,5 & 66,4 \\
\hline C,V,(\%) & 8,3 & 9,1 & 17,8 & 5,7 & 8,7 & 4,3 \\
\hline
\end{tabular}

PCv=Proteína cruda vainas; $\mathrm{PCh}=$ Proteína cruda hojas; $\mathrm{PCt}=$ Proteína cruda tallos, FNDv=Fibra neutro detergente en vainas; FNDh=Fibra neutro detergente en hojas; FNDt=Fibra neutro detergente en tallos. Medias con la misma letra en cada columna no difieren de acuerdo con la prueba de MDS al 5\%.

Cuadro 4. Medias de los cultivares para las variables rendimiento de biomasa, materia seca, producción de grano y días a R6.

\begin{tabular}{|c|c|c|c|c|c|}
\hline Cultivares & $\begin{array}{l}\text { Materia Verde } \\
\quad\left(\mathrm{kg} \mathrm{ha}^{-1}\right)\end{array}$ & $\begin{array}{c}\text { Materia Seca } \\
\quad\left(\mathrm{kg} \cdot \mathrm{ha}^{-1}\right)\end{array}$ & $\begin{array}{l}\text { Rend., grano } \\
\quad\left(\mathrm{kg} \cdot \mathrm{ha}^{-1}\right)\end{array}$ & $\begin{array}{c}\mathrm{V}: \mathrm{H}: \mathrm{T} \\
(\%)\end{array}$ & Días a R6 \\
\hline CIGRAS-06 & 52331,7 a & $17245,4 \mathrm{a}$ & $5013,1 \mathrm{a}$ & $33: 34: 33$ & $97,0 \mathrm{~d}$ \\
\hline FP92-1904 & 26742,9 def & 9317,7 cde & $2868,8 \mathrm{bc}$ & $21: 48: 31$ & $104,0 \mathrm{~b}$ \\
\hline FP93-1935 & $43888,5 \mathrm{ab}$ & 13074,6 b & $3121,9 \mathrm{bc}$ & $18: 50: 32$ & $100,7 \mathrm{c}$ \\
\hline FP90-7102 & 23087,8 ef & 8724,4 de & $2497,2 \mathrm{bc}$ & $23: 47: 30$ & $100,7 \mathrm{c}$ \\
\hline FP93-1924 & $38331,7 \mathrm{bc}$ & $13066,2 \mathrm{~b}$ & $3792,5 \mathrm{ab}$ & $23: 46: 32$ & $104,7 \mathrm{ab}$ \\
\hline FP90-10205 & 22112,5 ef & $7555,3 \mathrm{e}$ & $2933,8 \mathrm{bc}$ & $19: 47: 33$ & $105,0 \mathrm{ab}$ \\
\hline FP92-4906 & $16275,6 \mathrm{f}$ & $6816,0 \mathrm{e}$ & $1537,0 \mathrm{c}$ & $19: 53: 28$ & 106,0 a \\
\hline FP90-6103 & $30313,5 \mathrm{cde}$ & $10623,5 \mathrm{bcd}$ & $3593,3 \mathrm{ab}$ & $27: 40: 33$ & $100,3 \mathrm{c}$ \\
\hline FP93-1930 & 29354,3 cde & 9866,8 bcde & $2845,1 \mathrm{bc}$ & $22: 47: 31$ & $103,7 \mathrm{~b}$ \\
\hline FP90-6102 & $37739,5 \mathrm{bcd}$ & $12586,8 \mathrm{bc}$ & $3784,1 \mathrm{ab}$ & $24: 47: 29$ & $104,3 \mathrm{~b}$ \\
\hline Promedio & 29212,0 & 10888,0 & 3198,7 & $23: 46: 31$ & 102,6 \\
\hline $\mathrm{C}, \mathrm{V},(\%)$ & 26,4 & 21,6 & 22,0 & - & 0,8 \\
\hline
\end{tabular}

Medias con la misma letra en cada columna no difieren de acuerdo con la prueba de MDS al 5\%,

V:H:T=Proporción de la materia seca de vainas, hojas y tallos en la planta. 
y $5013 \mathrm{~kg} \cdot \mathrm{ha}^{-1}$, respectivamente. Estos valores reflejan el alto potencial del cultivar CIGRAS-06 como productor de forraje y grano.

Con respecto a los cultivares de la Fundación DANAC revisten interés los rendimientos en biomasa y materia seca de los germoplasmas FP93-1935, FP93-1924 y FP90-6102, con valores superiores a los 37 ton.ha ${ }^{-1}$ de materia verde y 12,5 ton.ha ${ }^{-1}$ de materia seca. Los cultivar FP93-1924, FP90-6102 y FP90-6103 se destaca por el alto rendimiento de grano similar al comportamiento del CIGRAS-06 con valores superiores a los $3500 \mathrm{~kg} \cdot \mathrm{ha}^{-1}$. Villalobos y Camacho (1999), en Costa Rica obtuvieron valores de materia seca en el rango del promedio de este experimento de 9 a 12 ton.ha $^{-1}$ con diferentes cultivares sembrados en fotoperiodos crecientes (Mayo) y de 5 a 7 ton.ha ${ }^{-1}$ cuando se sembraron en fotoperiodos decrecientes (Noviembre).

La proporción de materia seca de vainas, hojas y tallo (V:H:T) en los cultivares CIGRAS-06,
FP93-1935, FP93-1924 y FP90-6102 fueron de 33:34:33, 18:50:32, 23:47:30 у 24:47:29, respectivamente. Estas varían según el cultivar, radiación solar, edad de la planta, nivel de suplencia hídrica y el sistema de siembra empleado (Tobía y Villalobos 2004, Tobía 2011). Un atributo favorable del cultivar CIGRAS-06 es la mayor proporción de vainas en relación con el resto de los cultivares y es en esta fracción en donde se acumula la mayor cantidad de PC y grasas (Tobía y Villalobos 2004). Con la excepción del CIGRAS-06 todos los otros cultivares presentaron mayor proporción de hojas y menos de vainas, manteniéndose la de tallos entre 28 y $33 \%$ en todos los materiales.

De igual manera, en el Cuadro 4 se evidencia que el cultivar S-06 es el más precoz y logra alcanzar el estado $\mathrm{R}_{6}$ a los 97 días. El resto de los cultivares tardan entre 100 y 106 días.

En la Figura 1 se presenta la representación gráfica del análisis de componentes principales

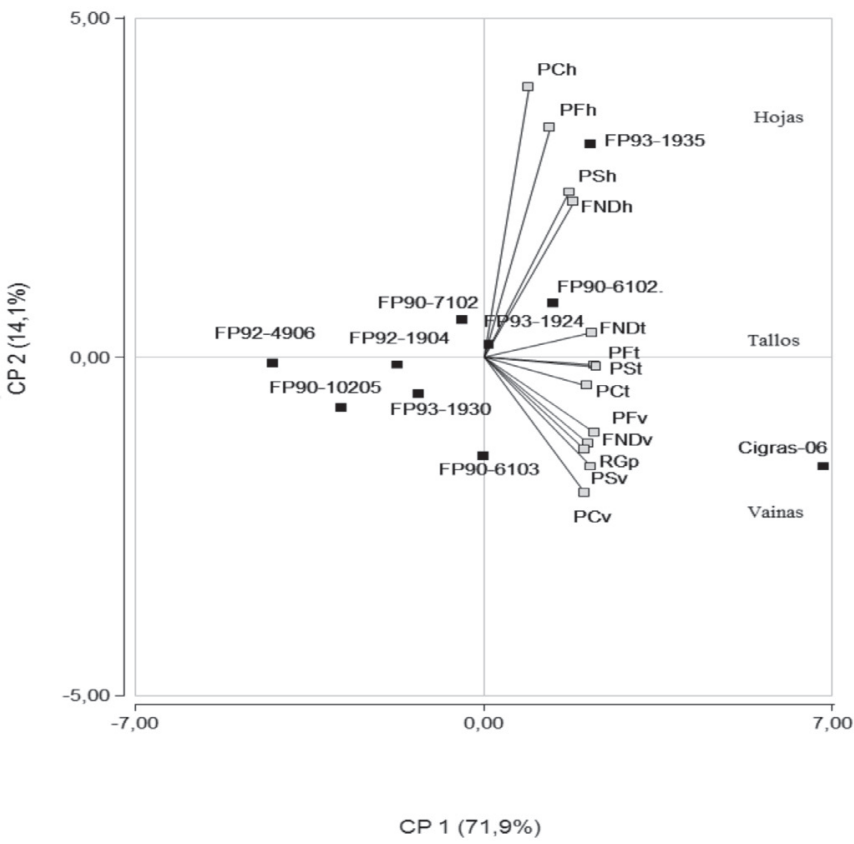

$\mathrm{RGp}=$ Rendimiento del grano por planta, MSp=Masa seca planta, MFp=Masa fresca planta, $\mathrm{PCv}=$ Proteína cruda vainas, PCh=Proteína cruda hojas, PCt=Proteína cruda tallos, PCp=Proteína cruda planta, FNDv=Fibra neutra detergente vainas, $\mathrm{FNDh}=$ Fibra neutra detergente hojas, FNDt=Fibra neutra detergente tallos.

Fig. 1. Representación gráfica del análisis de componentes principales (ACP) obtenida de la evaluación de los 10 cultivares de soya con fines de forraje. Vectores de variables en el sentido de la fracción que mayormente determina el rendimiento de biomasa. 
obtenido de la evaluación con fines de forraje. Este tipo de análisis multivariado, que explica el $86,0 \%$ de la variación observada, permitió una visión holística del comportamiento de los 10 cultivares. El componente $\mathrm{CP} 1$ es el más relevante y ubica en el cuadrante positivo a los cultivares con mejor comportamiento para forraje (CIGRAS-06, FP90-6102, FP90-6103, FP93-1924 y FP93-1935) por la orientación con las variables bromatológicas de calidad y cantidad de biomasa. Se puede relacionar el rendimiento de biomasa en el caso de CIGRAS-06 por su mayor proporción de vainas y para el cultivar FP93-1935 por su alta proporción de hojas.

El cultivar CIGRAS-06 mostró el mejor comportamiento como planta forrajera y granera que superó a todos los genotipos en altura, peso de 100 semillas, número de semillas por vainas, peso fresco de la parte aérea y rendimiento de grano, lo que demostró su alta capacidad de adaptación a las condiciones tropicales. Hintz y Albrecht (1994), señalan que la variedad mejor productora de semillas es también la mejor forrajera, debido a que en esta parte de la planta se concentra la materia seca más rica en proteínas y grasas.

\section{CONCLUSIONES}

Los cultivares promisorios como CIGRAS-06; FP93-1935, FP93-1924 y FP906102, identificados en este experimento, tienen un alto potencial para ser explotado como leguminosa forrajera y a su vez ser utilizados en la producción comercial de semillas mediante uso de las prácticas comúnmente empleadas para producir grano.

\section{LITERATURA CITADA}

ACEVEDO F. 1998. Rendimiento y otras características biométricas de cultivares de soya en Portuguesa, Venezuela. Revista Unellez de Ciencia y Tecnología 16(1):17-36.

A.O.A.C. 1990. Association of Official Analytical Chemistry. Official methods of analysis. Arlington. Virginia. $1298 \mathrm{p}$.
BALZARINI M., DI RIENZO J. 2012. InfoGen versión 2012. FCA, Universidad Nacional de Córdoba, Argentina. Consultado junio 2013 en http://www. info-gen.com.ar

FEDEAGRO. 2011. Estadísticas Agrícolas. Confederación Nacional de Asociaciones de Productores Agropecuarios. Consultado agosto 2013 http://www. fedeagro.org/detalle 5. asp?id=1458

FEHR W., CAVINESS C. 1980. Stages of soybean development. Special Report 80. Cooperative Extension Service, Iowa State University, Ames, Iowa 50011. p.11.

GAZZONI D. 1995. Botánica, pp. 1-12. In: EMBRAPACNPSo. El cultivo de la soya en los trópicos: Mejoramiento y producción. Colección FAO Nº. 27.

HARPER J.E. 1974. Soil and symbiotic nitrogen requirements for optimum soybean production. Crop Sci. 14:255-260.

HINTZ R., ALBRECHT K. 1994. Dry Matter partitioning and forage nutritive value of soybean plant component. Agronomy Journal 86:59-62.

JUÁREZ M. 2005. Influencia del manejo del riego en el crecimiento y producción de la soya (Glycyne max L. Merr) cultivar "CIGRAS-06" Trabajo Especial de Grado, Universidad Centroccidental "Lisandro Alvarado". Barquisimeto, Venezuela. 64 p.

MADURO A., ORTÍZ J., MIRANDA H., TOBÍA C., PEROZA D. 2012. Efecto de láminas de riego sobre el cultivo de la soya (Glycine max L.). Revista Unellez de Ciencias y Tecnología 30:11-18.

NORMAN A. 1983. Fisiología, mejoramiento, cultivo y utilización de la soya. Buenos Aires. Hemisferio Sur. 244 p.

RODRÍGUEZ R. 2003. Análisis de la información climatológica de la estación Miguel Luna Lugo del Decanato de Agronomía de la Universidad Centroccidental "Lisandro Alvarado" UCLA. Periodo 1976-2002. Trabajo de ascenso, Universidad Centroccidental Lisandro Alvarado, Barquisimeto, Venezuela. $108 \mathrm{p}$.

SEQUERA C. 2009. Efecto de cinco edades de corte en la biomasa, composición química, fraccionamiento proteico y degradabilidad ruminal in situ del forraje de soya (Glycine max L. Merr.) cv. CIGRAS 06. Tesis de maestría, Universidad Centroccidental "Lisandro Alvarado", Barquisimeto, Venezuela. 102 p.

SOLÓRZANO P., MUÑOZ J., GAMBOA M. 2005. El Cultivo de la Soya en Venezuela. Agroisleña. Maracay, Venezuela. 188 p.

TOBÍA C. 2004. Introducción del ensilaje de soya en un sistema de producción intensiva de leche en el trópico húmedo de Costa Rica. Tesis de doctorado. Universidad de Costa Rica, San José, Costa Rica. 120 p.

TOBÍA C. 2011. Efecto de cinco niveles de suplencia hídrica sobre la biomasa y la composición química del 
forraje de soya (Glycine max L. Merr.) cv. CIGRAS 06 UCR-UCLA. Trabajo de Ascenso, Universidad Centroccidental "Lisandro Alvarado", Barquisimeto, Venezuela. $100 \mathrm{p}$.

TOBÍA C., SEQUERA C., VILLALOBOS E., CIOFFI R., ESCOBAR O. 2007. Experiencias en la elaboración de silaje maíz-soya en dos sistemas de producción bovina en Venezuela, pp. 78-87. In: XI Seminario Manejo y Utilización de Pastos y Forrajes en Sistemas de Producción Animal (Memorias). Funda Pasto. UCLA. Barquisimeto, Venezuela.

TOBÍA C., VILLALOBOS E. 2004. Producción y valor nutricional del forraje de soya en condiciones tropicales adversas. Agronomía Costarricense 28(1):17-25.

TOBÍA C., VILlalobOS E., RICO E. 2006. Uso del forraje de soya (Glycine $\max$ L. Merr.) cv. CIGRAS 06 en la nutrición de rumiantes, pp. 77-86. In: X
Seminario Manejo y Utilización de Pastos y Forrajes en Sistemas de Producción Animal (Memorias). Funda Pasto. Universidad del Zulia. Maracaibo. Venezuela.

VAN SOEST P., ROBERTSON J., LEWIS B. 1991. Methods for dietary fiber, neutral detergent fiber and nonstarch polysaccharides in relation to animal nutrition. Journal of Dairy Science 74:3583-3591.

VILLALOBOS E., CAMACHO F. 1999. Avances en el mejoramiento genético de la soya en Costa Rica. II CIGRAS-06 y CIGRAS-10, dos nuevas variedades tropicales. Agronomía Costarricense 23(1):61-67.

VILLALOBOS E., CAMACHO F. 2003. Registration of 'CIGRAS-06' soybean. Crop Science 43:1122-1122.

WEISS W., SHOCKEY W. 1991. Value of orchard grass and alfalfa silage fed with varying amounts of concentrates for dairy cows. Journal of Dairy Science 74:1933-1943. 